\title{
Characterization of bubbly flow systems, A review
}

\begin{abstract}
Bubbly flows occur frequently in natural systems and are also used for different applications in energy-producing and chemical and petroleum industries. The characterization of bubbles is important in many chemical and petrochemical engineering applications. In such applications, the concentration, morphology and spatial distribution of bubbles determines the pressure drop and wall heat transfer. Past studies have reported different techniques for the characterization of these bubbles. Broadly, these can be characterized into two -intrusive and non-intrusive. The current paper presents a detailed review on both the intrusive and non-intrusive techniques which have been used for the characterization of the gas-liquid systems or gas-liquidsolid systems in general, and bubbly flows in particular.
\end{abstract}

Volume 2 Issue 6 - 2017

\author{
Ashish Karn, ${ }^{1,2}$ John Noonan' \\ 'St. Anthony Falls Laboratory, University of Minnesota, USA \\ ${ }^{2}$ Department of Mechanical Engineering, College of Engineering \\ Roorkee, India
}

Correspondence: Ashish Karn, Department of Mechanical Engineering, College of Engineering Roorkee, India, Tel + |-6I2642-1860,Email karn@umn.edu

Received: April 15, 2017| Published: July 03, 2017

Keywords: gas-liquid flows, fluidized bed, image analysis, intrusive techniques, non-intrusive techniques, bubble size, bubbly flows

Abbreviations BSD, bubble size distribution; PET, positron emission tomography; PIV, particle image velocimetry, RPT; radioactive particle tracking; NMR, nuclear magnetic resonance imaging

\section{Introduction}

Multiphase flow systems have been applied extensively in chemical, physical, petrochemical and biochemical processing industries. ${ }^{1-3}$ Among different multiphase flow systems, bubbly flow is a two phase flow where small bubbles are dispersed or suspended in a liquid continuum. Typical features of this flow are moving and deformable interfaces of bubbles in time and space domains and complex interactions between the interfaces, and also between the bubbles and the liquid flow. Bubbly flows occur frequently in natural systems and are also used for different applications in energy-producing and chemical and petroleum industries. Some of the common applications involve bubble columns which are used as reactors in a variety of chemical and biochemical processes, e.g. the Fischer-Tropsch process for hydrocarbon synthesis, hydrogenation of unsaturated oil, coal liquefaction, fermentation, waste water treatment etc. ${ }^{4,5}$ Bubbly flows are also ubiquitously found in flotation cells ${ }^{6}$ and spargers ${ }^{7}$ and have recently been investigated extensively for aeration studies. ${ }^{8-12}$ Concentration, size and velocity distributions of solid or liquid particles suspended in gas or liquid are important in many processes such as meteorological research, biology, environmental protection, chemistry, medicine and agricultural engineering. Further, commercial activities such as conversion of natural gas to fuels and chemicals have prompted further fundamental research in fluid and bubble dynamics, transport phenomena and the effects due to scale up of three-phase fluidization systems.

The prediction of pressure drop and wall heat transfer is necessary in many of these processes and these are strongly dependent upon the concentration, morphology and spatial distribution of the bubbles. ${ }^{13}$ Similarly, in many liquid-gas systems, gases are dispersed in liquids to obtain large interfacial area for chemical reactions, heat and mass transfer processes. The rate of these processes is determined by bubble surface area flux which is closely linked to the bubble size distribution. ${ }^{14}$

\section{Discussion}

Different techniques have been employed to measure bubble size distributions. Broadly, it can be divided into two categoriesintrusive and non-intrusive techniques. ${ }^{15}$ Both these methods have been extensively reported in the literature and a brief description of different intrusive and non-intrusive techniques is provided below,

\section{Intrusive techniques}

Considerable intrusive techniques have been developed to study bubble behavior in gas-liquid and gas-liquid-solid fluidized systems. These intrusive techniques include impedance (conductivity or resistivity) probes, capillary suction probes, ${ }^{16}$ optical fiber probes, ultrasound probes, endoscopic probes, wire-mesh sensors ${ }^{17}$ and hot film anemometry.

The impedance probe has been applied to measure the bubble volume fraction, bubble length and bubble rise velocity in three-phase fluidized beds utilizing the difference in conductivity between the liquid and the gas phase. ${ }^{18,19}$ Similarly, the dual electrical resistivity probe has been used to study the bubble size,,$^{20-22}$ bubble rise velocity $^{22-24}$ and gas volume..$^{21,24}$ To discern the local flow structures in fluidized beds, the conductivity probe signal can be analyzed based on the statistical, fractal, chaos and wavelet analyses. ${ }^{25}$ The optical fiber probe utilizes the principle that the light transmits in liquid medium and is reflected by the gas medium or bubbles and is effective when the difference in the refraction index between the gas and liquid phases is large. The optical fiber probe has been used to measure the gas volume fraction, ${ }^{26-28}$ bubble size, ${ }^{28,29}$ bubble frequency in a three-phase fluidized bed, ${ }^{26-28}$ directional distribution of bubble size, ${ }^{30,31}$ bubble rise velocity, ${ }^{28,29}$ direction of movement and shape of the bubble. ${ }^{32}$ The specific advantage for using the optic fiber probe technique lies in the fact that it can be effectively applied to high-pressure and hightemperature conditions for the bubble property measurement.

A slew of other intrusive techniques have been employed by researchers across the world for studying bubbly flows. For instance, the statistical analysis of acoustic signals was used by Al-Masry et al. ${ }^{33}$ to study the BSD and bubble frequency in bubble columns. These 
acoustic signals undergo significant attenuation due to reflection on gas bubbles and thus are unsuitable for use under high void fractions. ${ }^{34} \mathrm{~A}$ combination of imaging system with fiber optic probe and endoscopic probe has been used by some authors in liquid-solid or gas-solid systems. ${ }^{35,36}$ Dual-probe hot-film anemometry has been used by Wang \& Ching $^{37}$ to measure bubble velocities in the gas-liquid flow at low solids concentration conditions. Hot film anemometry is limited in its use because it can measure the properties only at low solids concentration and also in the case of uniform temperature distribution in the measured volume.

However, it is worth noting that the probe measures the point properties. The primary disadvantage for intrusive probe techniques is that the probe interferes with the flow field and the bubble dynamics. The immersed probe in the flow can cause disintegration, acceleration or elongation of the bubbles. ${ }^{38-42}$ Reduction in the size of the probe, although reduces the effect of the interference, but makes the probe susceptible to damage.

\section{Non-intrusive techniques}

Contrary to the intrusive technique, the non-intrusive technique has the advantage of no measurement interference with the flow field. The non-intrusive techniques have been used for a variety of information including the cross-sectional bed density profile to the particle trajectory map. ${ }^{43-46}$ Some of the non-intrusive techniques, typically used to measure the three-phase fluidized bed properties include X-ray, $\gamma$-ray, positron emission tomography (PET), pressure transducer, visualization techniques, particle image Velocimetry (PIV), radioactive particle tracking (RPT), ultrasonic tomography, nuclear magnetic resonance imaging (NMR), laser techniques and electrical tomography. Some measurement examples in three-phase fluidized beds are described below.

To study the bubble flow behavior in the bubble column and threephase fluidized beds, pressure drop measurement is coupled with a statistical analysis technique. ${ }^{47-51}$ The pressure fluctuation signals in these transducers represent the overall hydrodynamic behavior in the bubble column. In other words, different bubble phenomena in the flow such as bubble coalescence and bubble breakup, bubble burst at the top surface, and bubble formation at the distributor contribute to these signals. The X-ray technique is another important non-intrusive technique that has been widely used to investigate the bubble flow behavior such as bubble size and shape, bubble rise velocity, bubble growth and bubble breakage (see). ${ }^{52}$ The X-ray technique consists of the X-ray source to generate the X-ray beam, an image intensifier to produce an image, a CCD video camera to capture the image, and the image analysis system. Another modification of this technique is the X-ray computer assisted tomography (CAT) that provides a high spatial resolution, was developed by Kumar et al., ${ }^{53}$ and have been used subsequently to measure the phase holdups. ${ }^{45}$ Similarly, the $\gamma$-ray density gauge technique has been employed to study the bubble size, bubble frequency and bubble coalescence. In this technique, the voidage between the radiation source and detector in the bed is obtained by relating the ionization of gas to the amount of radiation received by the detector. It has been used to study the jet and bubble behavior, voidage distribution in the bubble column, gas holdups in the prior studies. ${ }^{54-57}$ It should be noted that owing to its long scanning time, this technique is suited for studying the time-averaged flow properties only, and not for the study of bubble formation and bubble dynamics in the bed.
Non-intrusive laser techniques are also widely used to study bubble behavior, including the PIV, laser Doppler anemometry (LDA), phase Doppler anemometry (PDA) and laser Doppler Velocimetry (LDV). Several authors have used these techniques to investigate the bubble characteristics such as bubble size distribution, ${ }^{58-60}$ liquid velocity and turbulence, ${ }^{61}$ velocity of liquid and bubbles ${ }^{62}$ etc. For all the abovementioned laser techniques, the laser beam needs to penetrate the flow system and are thus limited only to the low gas holdup conditions.

Some other non-intrusive techniques are used for tracking the particle movement, and/or mapping the instantaneous or timeaveraged, local or cross-sectional averaged, phase holdups and phase velocities. These include PET, RPT, ultrasonic tomography, nuclear magnetic resonance imaging, electrical impedance tomography and electrical capacitance tomography. Another interesting technique involves direct visualization with digital image analysis. It offers many advantages in terms of flexibility, relative insensitivity to the optical properties of the dispersed phase, easier optics alignment as compared to laser-diffraction methods, as well as the capability of providing the velocity and size information of the dispersed phase simultaneously. The optical image analyses have been used recently for quantifying the bubble size distribution. ${ }^{63-66}$ Luo et al.$^{67}$ utilized the visualization technique to study the bubble characteristics and bubble formation behavior. A number of studies have been recently conducted using this approach with different algorithms for image analysis. ${ }^{8,65,66,68-74}$ Although it is very convenient and time efficient for online monitoring and analysis of a large number of images, to implement this technique for real-time analysis of the bubbly flow images from different engineering applications pose multifarious challenges. Generally, due to the excessive coalescence and breakup of bubbles, most of the proposed techniques for bubble image processing produce significant errors when applied to flows with high gas and liquid velocities. These errors are closely related to the challenge of extracting accurate bubble information from large clusters due to the coalescence of bubbles. Overall, these techniques are still limited in their robustness to resolve large bubble clusters particularly under highly unsteady flows with large void fractions of bubbles. Another limitation of these techniques is related to their ability to deal with a wide range of bubble size distribution. In addition, algorithms with significant improvements in computational speed are needed for fast processing of a large number of images and online monitoring of bubble concentration and distribution.

\section{Conclusion}

Multiphase flows, particularly bubbly flows, are important in many industrial applications particularly chemical and petrochemical processing industries. Some of the common applications include bubble columns, flotation cells, spargers and fluidized beds. In many of these applications, the concentration, size and velocity of these bubbles suspended in liquid are important. The prediction of heat and mass transfer rates, pressure drop etc. is important for these applications. In the current review paper, different kinds of techniques for the characterization of these bubbles are discussed. First, a detailed account of the intrusive techniques is presented. Different intrusive techniques are discussed including impedance probe, electrical resistivity probe, optical fiber probe, hot film anemometry etc. It is noted that the major limitation of using the intrusive technique is the interference of the probe with the flow field. Thus, non-intrusive techniques are preferred over intrusive techniques. Then, a description of non-intrusive techniques are presented including x-ray technique, 
$\gamma$-ray density gauge technique, laser techniques like LDV, PIV, PDA etc. Finally, a description of image analysis technique for the study of bubbly flows is suggested. However, the image analysis has its own limitations, particularly in flows with high void-fraction.

\section{Acknowledgements}

None.

\section{Conflict of interest}

The author declares no conflict of interest.

\section{References}

1. Shah, Yatish T. Gas-liquid-solid reactor design. New York: McGrawHill; 1979. 60 p.

2. Ramachandran PA, RV Chaudhari. Three-Phase Catalytic Reactors. New York, USA: Gordon and Breach; 1983. 169 p.

3. Fan, Liang-Shih. Gas-liquid-solid fluidization engineering. Butterworth. Stoneham, MA. 1989

4. Smith, Jeffrey S, Louis F Burns, et al. Bubble column reactors for wastewater treatment. 2. The effect of sparger design on sublation column hydrodynamics in the homogeneous flow regime. Industrial \& engineering chemistry research. 1996;35(5):1700-1710.

5. Lau YM, NG Deen, JAM Kuipers. Development of an image measurement technique for size distribution in dense bubbly flows. Chemical Engineering Science. 2013;94:20-29.

6. Sadr-Kazemi N, JJ Cilliers. An image processing algorithm for measurement of flotation froth bubble size and shape distributions. Minerals Engineering. 1997;10(10):1075-1083.

7. Geary, Nicholas W, Richard G Rice. Bubble size prediction for rigid and flexible spargers. AIChE journal. 1991;37(2):161-168.

8. Karn A, Christopher Ellis, Roger Arndt, et al. An integrative image measurement technique for dense bubbly flows with a wide size distribution. Chemical Engineering Science. 2015;122:240-249.

9. Karn A, Christopher Ellis, Jiarong Hong, et al. Investigations into the turbulent bubbly wake of a ventilated hydrofoil: Moving toward improved turbine aeration techniques. Experimental Thermal and Fluid Science. 2015;64:186-195.

10. Karn A, Garrett M, Monson, et al. Mass transfer studies across ventilated hydrofoils: A step towards hydroturbine aeration. International Journal of Heat and Mass Transfer. 2015;87:512-520.

11. Karn A, Christopher R Ellis, Christopher Milliren, et al. Bubble size characteristics in the wake of ventilated hydrofoils with two aeration configurations. International Journal of Fluid Machinery and Systems. 2015;8(2):73-83.

12. Karn A, Siyao Shao, Roger EA Arndt, et al. Bubble coalescence and breakup in turbulent bubbly wake of a ventilated hydrofoil. Experimental Thermal and Fluid Science. 2016;0:397-407.

13. Junker B. Measurement of bubble and pellet size distributions: past and current image analysis technology. Bioprocess and biosystems engineering. 2006;29(3):185-206

14. Yang GQ, Bing Du, LS Fan. Bubble formation and dynamics in gasliquid-solid fluidization-A review. Chemical Engineering Science. 2007;62(1):2-27.

15. Kamp AM, AK Chesters, C Colin, et al. Bubble coalescence in turbulent flows:a mechanistic model for turbulence-induced coalescence applied to microgravity bubbly pipe flow. International Journal of Multiphase Flow. 2001;27(8):1363-1396.
16. Laakkonen MP, Moilanen T, Miettinen K, et al. Local bubble size distributions in agitated vessel:comparison of three experimental techniques. Chemical Engineering Research and Design. 2005;83(1):50-58.

17. Prasser HM. Novel experimental measuring techniques required to provide data for CFD validation. Nuclear Engineering and Design. 2008;238(3):744-770.

18. Hills JH, RC Darton. The rising velocity of a large bubble in a bubble swarm. Trans Inst Chem Eng. 1976;54(4):258-264.

19. Boyer, Christophe, Anne-Marie Duquenne, et al. Measuring techniques in gas-liquid and gas-liquid-solid reactors. Chemical Engineering Science. 2002;57(16):3185-3215.

20. Matsuura Akinori, Liang-Shih Fan. Distribution of bubble properties in a gas-liquid-solid fluidized bed. AICHE journal. 1984;30(6):894-903.

21. Tang WT, Liang-Shih Fan. Hydrodynamics of a three-phase fluidized bed containing low-density particles. AIChE journal. 1989;35(3):355-364.

22. Liu TJ. Bubble size and entrance length effects on void development in a vertical channel. International Journal of Multiphase Flow. 1993;19(1):99-113.

23. Chen Z, C Zheng, Y Feng, et al. Local bubble behavior in three-phase fluidized beds. The Canadian Journal of Chemical Engineering. 1998;76(2):315-318.

24. Zenit Roberto, Donald L Koch, Ashok S Sangani. Measurements of the average properties of a suspension of bubbles rising in a vertical channel. Journal of Fluid Mechanics. 2001;429:307-342.

25. Briens Lauren A, Naoko Ellis. Hydrodynamics of three-phase fluidized bed systems examined by statistical, fractal, chaos and wavelet analysis methods. Chemical engineering science. 2005;60(22):6094-6106.

26. Lee SL, HI de Lasa, MA Bergougnou. A U-shaped fiber optic probe to study three-phase fluidized beds. Particulate Science and Technology. 1986;4(1):61-71.

27. Lee SLP, HI De Lasa. Phase holdups in three-phase fluidized beds. $A I-$ ChE journal. 1987;33(8):1359-1370.

28. Shoukri Mamdouh, Ibrahim Hassan, Ihab Gerges. Two-phase bubbly flow structure in large-diameter vertical pipes. The Canadian Journal of Chemical Engineering. 2003;81(2):205-211.

29. Xue Junli, Muthanna Al-Dahhan, Milorad P, et al. Bubble Dynamics Measurements Using Four-Point Optical Probe. The Canadian Journal of Chemical Engineering. 2003;81(3-4):375-381.

30. Yu Yong Ho, Sang D Kim. Bubble characteristics in the radial direction of three-phase fluidized beds. AIChE journal. 1988;34(12):2069-2072.

31. Chabot Julie, Hugo I De Lasa. Gas holdups and bubble characteristics in a bubble column operated at high temperature. Industrial and Engineering Chemistry Research. 1993;32(11):2595-2601.

32. Frijlink Jacob Jan. Physical aspects of gassed suspension reactors. 1987.

33. Al-Masry WA, Emad M Ali, Yhya M Aqeel. Determination of bubble characteristics in bubble columns using statistical analysis of acoustic sound measurements. Chemical Engineering Research and Design. 2005;83(10):1196-1207.

34. Bröring S, J Fischer, T Korte, et al. Flow structure of the dispersed gasphase in real multiphase chemical reactors investigated by a new ultrasound-doppler technique. The Canadian Journal of Chemical Engineering. 1991;69(6):1247-1256.

35. Peters Michael H, Liang-Shih Fan, Thomas L Sweeney. Study of particle ejections in the freeboard region of a fluidized bed with an image carrying probe. Chemical Engineering Science. 1983;38(3):481-485. 
36. Kumar Samir K, Kusakabe K, Raghunathan, et al. Mechanism of heat transfer in bubbly liquid and liquid-solid systems: Single bubble injection. AIChE journal. 1992;38(5):733-741.

37. Wang G, CY Ching. Measurement of multiple gas-bubble velocities in gas-liquid flows using hot-film anemometry. Experiments in fluids. 2001. 31(4):428-439.

38. Rowe PN. Interaction of bubbles with probes in gas fluidized beds. Trans. IChemE. 1981;59:177.

39. Chabot J, SLP Lee, A Soria, et al. Interaction between bubbles and fibre optic probes in a bubble column. The Canadian Journal of Chemical Engineering. 1992;70(1):61-68.

40. Kiambi SL, Anne-Marie Duquenne, JB Dupont, et al. Measurements of bubble characteristics:comparison between double optical probe and imaging. The Canadian Journal of Chemical Engineering. 2003;81(3-4):764-770

41. Zenit R, DL Koch, AS Sangani. Impedance probe to measure local gas volume fraction and bubble velocity in a bubbly liquid. Review of Scientific Instruments. 2003;74(5):2817-2827.

42. Enrique Juliá J, Wouter K Harteveld, Robert F Mudde, et al. On the accuracy of the void fraction measurements using optical probes in bubbly flows. Review of scientific instruments. 2005;76(3):035103.

43. Chaouki Jamal, Faical Larachi, Milorad P Duduković. Noninvasive tomographic and velocimetric monitoring of multiphase flows. Industrial \& engineering chemistry research. 1997;36(11):4476-4503.

44. Seeger A, U Kertzscher, K Affeld, et al. Measurement of the local velocity of the solid phase and the local solid hold-up in a three-phase flow by X-ray based particle tracking velocimetry (XPTV). Chemical Engineering Science. 2003;58(9):1721-1729.

45. Hubers Jeremy L, Alex C Striegel, Theodore J Heindel, et al. X-ray computed tomography in large bubble columns. Chemical Engineering Science. 2005;60(22):6124-6133.

46. Warsito W, LS Fan. Dynamics of spiral bubble plume motion in the entrance region of bubble columns and three-phase fluidized beds using $3 \mathrm{D}$ ECT. Chemical engineering science. 2005;60(22):6073-6084.

47. Drahoš J, F Bradka, M Punčochář. Fractal behaviour of pressure fluctuations in a bubble column. Chemical engineering science. 1992;47(1516):4069-4075.

48. Drahoš J, J Zahradnik, M Punčochář, et al. Effect of operating conditions on the characteristics of pressure fluctuations in a bubble column. Chemical Engineering and Processing: Process Intensification 1991;29(2):107-115.

49. Johnsson Filip, RC Zijerveld, JC Schouten, et al. Characterization of fluidization regimes by time-series analysis of pressure fluctuations. International journal of multiphase flow. 2000;26(4):663-715

50. Kluytmans, Jeroen HJ, Berend GM, et al. Gas holdup in a slurry bubble column:influence of electrolyte and carbon particles. Industrial \& engineering chemistry research. 2001;40(23):5326-5333.

51. Chilekar VP, MJF Warnier, J Van Der Schaaf, et al. Bubble size estimation in slurry bubble columns from pressure fluctuations. AIChE journal. 2005;51(7):1924-1937.

52. Fournier Th, Ch Jeandey. Optimization of an experimental setup for void fraction determination by the X-ray attenuation technique. In Measuring techniques in gas-liquid two-phase flows, Springer. 1984. p.199-228.

53. Kumar SB, MP Dudukovic, BA Toseland. Measurement techniques for local and global fluid dynamic quantities in two and three phase systems. Non-Invasive Monitoring of Multiphase Flows. 1997;1-45.

54. Seville JPK, JEP Morgan, R Clift. Tomographic determination of the voidage structure of gas fluidised beds in the jet region. In Proceedings of fluidization. 1986;87-94.
55. Kumar Sailesh B, Davood Moslemian, Milorad P Duduković. A $\gamma$-ray tomographic scanner for imaging voidage distribution in two-phase flow systems. Flow Measurement and Instrumentation. 1995;6(1):61-73.

56. Veera U Parasu, JB Joshi. Measurement of gas hold-up profiles in bubble column by gamma ray tomography: effect of liquid phase properties. Chemical engineering research and design. 2000;78(3):425-434.

57. Jin Haibo, Suohe Yang, Zhiwu Guo, et al. The axial distribution of holdups in an industrial-scale bubble column with evaluated pressure using $\gamma$-ray attenuation approach. Chemical Engineering Journal. 2005;115(1):45-50.

58. Braeske Heiko, Günter Brenn, Joachim Domnick, et al. Extended PhaseDoppler Anemometry for Measurements in Three-Phase Flows. Chemical engineering \& technology. 1998;21(5):415-420.

59. Lee DJ, X Luo, LS Fan. Gas disengagement technique in a slurry bubble column operated in the coalesced bubble regime. Chemical engineering science. 1999;54(13):2227-2236.

60. Kulkarni, Amol A, Jyeshtharaj B Joshi, et al. Determination of bubble size distributions in bubble columns using LDA. AIChE journal. 2004;50(12):3068-3084

61. Vial Ch, R Laine, S Poncin, et al. Influence of gas distribution and regime transitions on liquid velocity and turbulence in a 3-D bubble column Chemical engineering science. 2001;56(3):1085-1093.

62. Brenn Günter, Heiko Braeske, Franz Durst. Investigation of the unsteady two-phase flow with small bubbles in a model bubble column using phase-Doppler anemometry. Chemical engineering science. 2002;57(24):5143-5159.

63. Do Amaral CEF, RF Alves, MJ Da Silva, et al. Image processing techniques for high-speed videometry in horizontal two-phase slug flows. Flow Measurement and Instrumentation. 2013;33:257-264.

64. Ferreira A, G Pereira JA, Teixeira, et al. Statistical tool combined with image analysis to characterize hydrodynamics and mass transfer in a bubble column. Chemical engineering journal. 2012;180:216-228.

65. Kracht Willy, Xavier Emery, C Paredes. A stochastic approach for measuring bubble size distribution via image analysis. International Journal of Mineral Processing. 2013;121:6-11.

66. Lau YM, NG Deen, JAM Kuipers. Development of an image measurement technique for size distribution in dense bubbly flows. Chemical Engineering Science. 2013;94:20-29.

67. Luo Xukun, Guoqiang Yang, DJ Lee, et al. Single bubble formation in high pressure liquid-solid suspensions. Powder technology. 1998;100(23):103-112.

68. Ma Ye, Guanxi Yan, Alexander Scheuermann, et al. Size distribution measurement for densely binding bubbles via image analysis. Experiments in fluids. 2014;55(12):1860.

69. Ahmed Fadha Shakir, Brent A Sensenich, Saba A Gheni, et al. Bubble dynamics in 2D bubble column:comparison between high-speed camera imaging analysis and 4-point optical probe. Chemical Engineering Communications. 2015;202(1):85-95.

70. Amankwah Anthony, Chris Aldrich. Automatic estimation of bubble size distributions in flotation froths by use of a mean shift algorithm and watershed transforms. In Geoscience and Remote Sensing Symposium (IGARSS), IEEE. 2014. p.1608-1611.

71. Olaofe OO, KA Buist, NG Deen, et al. Improved digital image analysis technique for the evaluation of segregation in pseudo-2D beds. Powder technology. 2013;244:61-74.

72. Sathe Mayur J, Channamallikarjun S Mathpati, Sagar S Deshpande, et al. Investigation of flow structures and transport phenomena in bubble columns using particle image Velocimetry and miniature pressure sensors Chemical engineering science. 2011;66(14):3087-3107. 
73. Zhang Wen Hui, Xiaoya Jiang, Yin-Mingzi Liu. A method for recognizing overlapping elliptical bubbles in bubble image. Pattern Recognition Letters. 2012;33(12):1543-1548.
74. Taboada Blanca, L Vega-Alvarado, MS Cordova-Aguilar, et al. Semiautomatic image analysis methodology for the segmentation of bubbles and drops in complex dispersions occurring in bioreactors. Experiments in fluids. 2006;41(3):383-392. 